\title{
Sensorimotor learning deficits observed in children with
}

\section{sports-related concussion [version 1; peer review: 1 not}

\section{approved]}

\author{
Jinsung Wang ${ }^{1}$, Danny Thomas², Young Ik $\mathrm{Cho}^{3}$ \\ ${ }^{1}$ Department of Kinesiology, University of Wisconsin- Milwaukee, Milwaukee, WI, 53201, USA \\ ${ }^{2}$ Department of Pediatrics, Medical College of Wisconsin, Milwaukee, WI, 53226, USA \\ 3Joseph J. Zilber School of Public Health, University of Wisconsin- Milwaukee, Milwaukee, WI, 53201, USA
}

V1 First published: 05 Jun 2015, 4:143
https://doi.org/10.12688/f1000research.6575.1

Latest published: 23 Jul 2015, 4:143

https://doi.org/10.12688/f1000research.6575.2

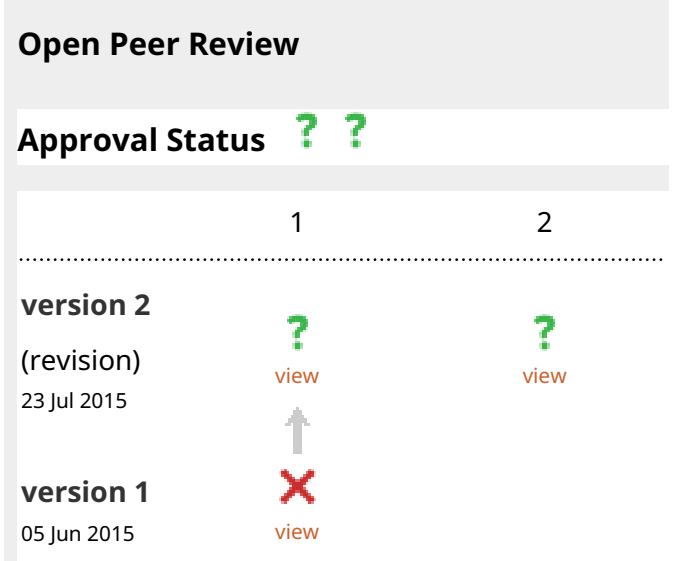

1. Lauren Sergio, York University, Toronto, USA

2. Liana Brown, Trent University,

Peterborough, Canada

Any reports and responses or comments on the article can be found at the end of the article. 
Corresponding author: Jinsung Wang (wang34@uwm.edu)

Competing interests: No competing interests were disclosed.

Grant information: The author(s) declared that no grants were involved in supporting this work.

Copyright: @ 2015 Wang J et al. This is an open access article distributed under the terms of the Creative Commons Attribution License, which permits unrestricted use, distribution, and reproduction in any medium, provided the original work is properly cited. Data associated with the article are available under the terms of the Creative Commons Zero "No rights reserved" data waiver (CC0 1.0 Public domain dedication).

How to cite this article: Wang J, Thomas D and Cho YI. Sensorimotor learning deficits observed in children with sports-related concussion [version 1; peer review: 1 not approved] F1000Research 2015, 4:143 https://doi.org/10.12688/f1000research.6575.1

First published: 05 Jun 2015, 4:143 https://doi.org/10.12688/f1000research.6575.1 


\section{Introduction}

Pediatric concussions have been shown to negatively affect neurocognitive function, including poor attention span, impaired memory and learning difficulties ${ }^{1-3}$. However, the association between pediatric concussion and sensorimotor function has not been investigated. Understanding motor learning impairment following sport-related concussion in children may be critical in guiding safe return to play. In this research, we investigated the pattern of adaptation to a novel visuomotor condition in three children who suffered a concussion while playing sports, and compared their adaptation patterns to those obtained from three children without a concussion. A research paradigm in which individuals adapt to a novel visuomotor condition during reaching movements has been employed extensively in the neuroscience community to understand the neural processes that underlie motor learning in both adults ${ }^{4,5}$ and children ${ }^{6,7}$.

We also examined neurocognitive function of both the concussed children and the control children using an assessment technique called the trail making test (TMT). TMT (part B) is a traditional paper assessment that times subjects as they draw lines to connect numbers and letters alternately on a page. TMT is a validated assessment of concussion, and tests cognitive demands that are also important for sports, including psychomotor processing, visual motor/spatial abilities and mental flexibility ${ }^{8,9}$.

The purpose of this observational study was to determine whether concussed children with neurocognitive impairments, as indicated by the TMT scores, would also demonstrate sensorimotor deficits, as indicated by the visuomotor adaptation patterns.

\section{Methods}

Three children (15 years old), who presented to the Emergency Department at the Children's Hospital of Wisconsin within 24 hours from the time of injury and who received a diagnosis of concussion (Glasgow Coma Scale $\geq 14$ ), participated in this study. They visited the Neuromechanics Laboratories at the University of Wisconsin-Milwaukee (UWM) 5 8 days following the concussion. Three children (12, 14 and 17 years old), who were recruited from the Milwaukee Metropolitan area, served as controls. Selection criteria for subjects were the same between patients and controls (except their concussion status), which were: subject is 10-17 years of age, regularly participates in an athletic activity, is English-speaking, is right handed, and has no neurological disease or peripheral disorder affecting movement of the right arm. All subjects were recruited and tested in May 2015.

Upon arrival at UWM, the subjects were first administered with the $\mathrm{TMT}^{8,9}$. Following that, they participated in the visuomotor adaptation experiment in which they performed rapid reaching movements from a start circle to a target repeatedly under a normal visuomotor condition (baseline) first, then under a novel visuomotor condition (adaptation). The baseline session (40 trials) was provided for the subjects to become familiarized with the general reaching task with unperturbed visual feedback. In the adaptation session (80 trials), the visual display of reaching movements was rotated 30 degrees counterclockwise about the start circle, such that a hand movement made in the "12 o'clock" direction resulted in a cursor movement made in the "11 o'clock" direction. Continuous visual feedback (in the form of a cursor) was provided throughout the movement in both sessions. A robotic exoskeleton called KINARM (BKIN Technologies Ltd, Kingston, ON, Canada) was used to provide the visuomotor rotation during the experiment and also to collect movement data. The 2-D position of arm segments was sampled at $1,000 \mathrm{~Hz}$, low-pass filtered at $15 \mathrm{~Hz}$, and differentiated to yield resultant velocity values. Data were processed and analyzed using MATLAB (The Mathworks Inc., Natick, MA) and SPSS.

To examine performance accuracy, we calculated direction error (DE), which was the angular difference between a vector from the start circle to the target and another vector from the hand position at movement start to that at peak arm velocity. Using the direction error data, we obtained the following measures for each subject: (1) DE at trial 1 in the adaptation session; (2) the first block of DE (i.e., mean of five consecutive trials) in the adaptation session that is not statistically different from the last block of DE in the baseline session; and (3) the rate of performance change during the adaptation session. To obtain the second measure, a priori pairwise comparisons, using t-tests, were made between DE at block 8 from the baseline session and DE at each of the 16 blocks from the adaptation session (starting from block 1). The alpha level was set at .05. To obtain the third measure, a line of approximation was constructed by fitting a logarithmic regression line to the adaptation data, and the slope value was used.

\section{Ethics}

All subjects and their parents signed the assent/consent forms approved by the Institutional Review Board of UWM (IRB\# 15.172).

\section{Sample size}

Because of the nature of the present study (i.e., observational/case study of concussed children), we only tried to recruit a small number of concussed children. We recruited all our subjects (three concussed children and three controls who met our selection criteria) within a 10-day window.

\section{Results}

Figure 1c illustrates neurocognitive data from all subjects, indicated by TMT scores. One concussed child (cc1) showed some cognitive slowing on visit 1 , as compared with the controls, while the others did not.

The overall pattern of visuomotor adaptation, illustrated in Figure 1a and b, was somewhat similar between the patients and the controls. In fact, the patients' baseline performances do not appear to be worse than those of the controls. However, a close examination of the adaptation data revealed the following differences:

1. DE at trial 1 of the adaptation session was somewhat larger for the patients than the controls; and within-group variability for this measure was also larger for the patients (Figure 1d).

2. The first block of DE in the adaptation session that was not statistically different from the last block of DE in the baseline session was observed much later in two of the patients than in the controls; and within-group variability for this measure was much larger for the patients (Figure 1b,e).

3. As indicated by the rate of performance change, it took longer for the patients than the controls to adapt to the visuomotor rotation; and within-group variability for this measure was slightly larger for the patients (Figure 1f). 

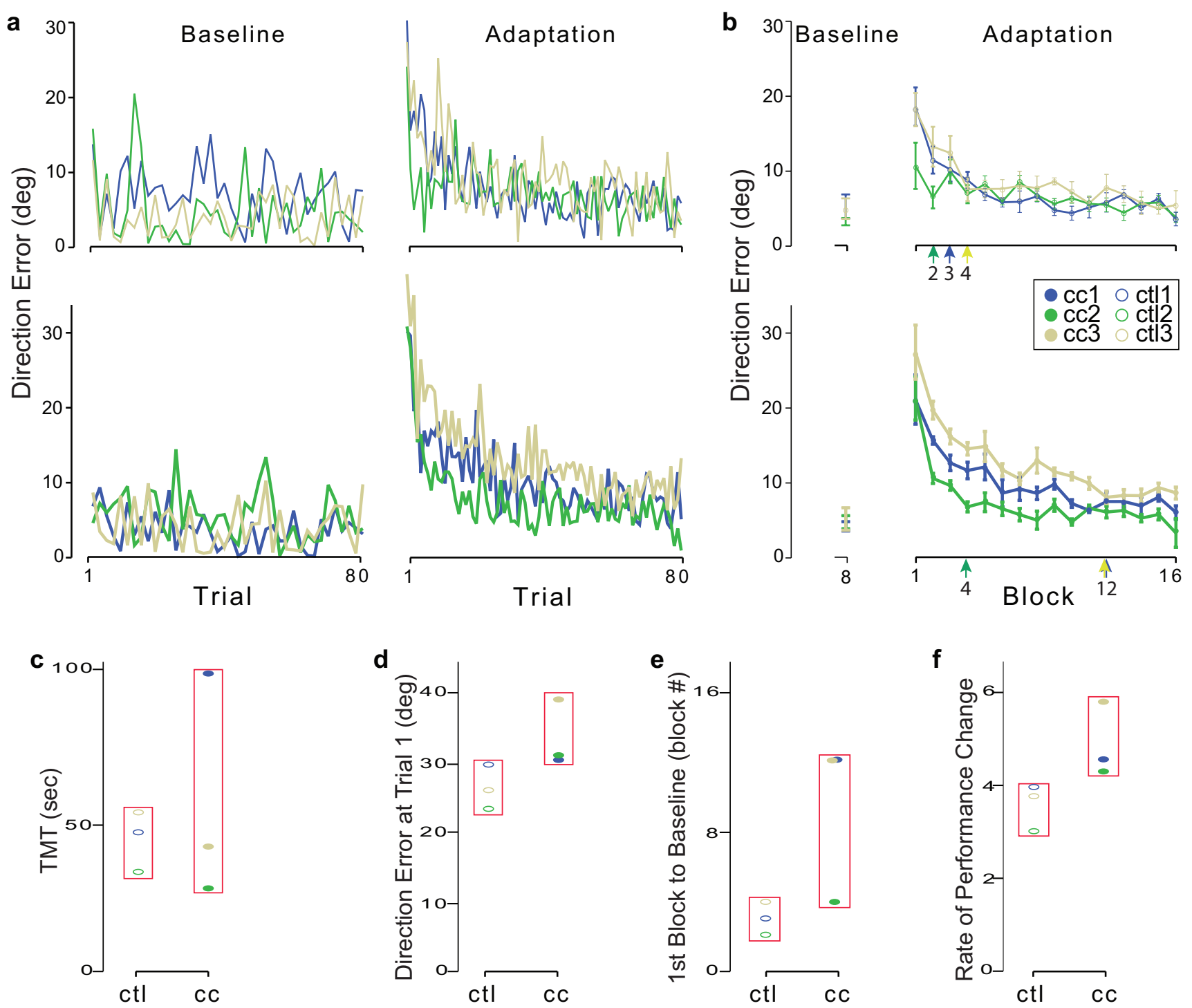

Figure 1. (a) Improvements in performance across trials during baseline and adaptation sessions. Upper panel depicts data from 3 controls, lower panel data from 3 concussed children. (b) Improvements in performance across blocks during the baseline (last block only) and adaptation sessions. Numbers under arrows indicate first block of DE that was not statistically different from last block of DE from the baseline phase. (c) TMT scores for every subject. (d) DE at trial 1 during the adaptation session. (d) First block of DE in the adaptation session that was not different from last block of DE in the baseline session. (e) Rate of performance change in the adaptation session. Vertical boxes in red $(\mathbf{c}-\mathbf{f})$ indicate variability within each subject group.

\section{Dataset 1. Kinematic data from visuomotor adaptation task}

http://dx.doi.org/10.5256/f1000research.6575.d48984

This SPSS file contains kinematic data from 3 subjects in each of the two groups (CC indicating concussed children, CTL controls). Subjects (ss) experienced two experimental sessions (1 indicating baseline, 2 visuomotor adaptation condition). To compute mean direction errors (dir_err) across 5 consecutive trials, the numbers shown in column 'trial' were converted into the numbers shown in column 'block'. The numbers shown in column 'block 2' were used to make statistical comparisons between mean direction error from the last block of baseline session and that from each of 16 blocks of adaptation session within each subject. Rot_ang indicates the degree to which the visual display was rotated ${ }^{12}$.

\section{Discussion}

TMT is a validated neurocognitive assessment of pediatric concussion $^{8,9}$. The average time to complete the TMT part B is $75 \mathrm{sec}-$ onds in neurologically intact individuals; if the time is longer than 273 seconds, it is considered deficient ${ }^{10,11}$. One concussed child who participated in our study completed the TMT part B in 98 seconds; and the other two children completed it in less than 50 seconds. According to the TMT scores, thus, one may conclude that all these children who had a concussion 5 to 8 days prior to their participation in this study did not seem to have neurocognitive impairments.

Their patterns of visuomotor adaptation observed in the patients, however, appear to be somewhat different from those observed in the controls. Specifically, the first block of DE in the adaptation session 
that is significantly different from the last block of DE of the baseline session occurred later in the patients than in the controls; and the rate of performance change was higher (i.e., slower adaptation) in the patients as well. These data indicate that the patients had more difficulty than the controls while adapting to the novel visuomotor rotation, which points to the possibility of sensorimotor learning deficits in the concussed children.

The present study has several limitations. First, all the children tested in this study were recruited in the Milwaukee Metropolitan area, which raises a possibility that they may not be the best representative of all children of the same age group. Also, we did not collect from our subjects any information regarding their demographic and social status, which could be considered as potential confounding characteristics. Finally, we did not conduct statistical analyses to quantify the differences between the children with concussion and those without concussion, because the statistical results would not be very meaningful given the small sample size. Nevertheless, our data exhibited qualitative differences between the two groups of children that suggest sensorimotor deficits in concussed children. Further investigation is warranted to demonstrate quantitative differences between children with and children without concussion by utilizing a larger sample size.

In conclusion, the results from this study indicate that children with concussion may have sensorimotor impairments even when they do not seem to have neurocognitive impairments. Given the importance of children's ability to rapidly adapt to the dynamic environments and task requirements throughout a sport, our findings suggest that examination of sensorimotor function, in addition to that of neurocognitive function, may provide valuable information when determining whether children are ready to return to play sports or not.

\section{Data availability}

F1000Research: Dataset 1. Kinematic data from visuomotor adaptation task, 10.5256/f1000research.6575.d48984 ${ }^{12}$

\section{Author contributions}

JW, DGT and YIC conceived the study and designed the experiment. JW carried out the research and prepared the first draft of the manuscript. DGT and YIC edited the manuscript. All authors have agreed to the final content of the manuscript.

\section{Competing interests}

No competing interests were disclosed.

\section{Grant information}

The author(s) declared that no grants were involved in supporting this work.
1. Browne GJ, Lam LT: Concussive head injury in children and adolescents related to sports and other leisure physical activities. Br J Sports Med. 2006; 40(2): 163-8.

PubMed Abstract | Publisher Full Text | Free Full Text

2. Thomas DG, Collins MW, Saladino RA, et al:: Identifying neurocognitive deficits in adolescents following concussion. Acad Emerg Med. 2011; 18(3): 246-54. PubMed Abstract | Publisher Full Text | Free Full Text

3. Sandel NK, Lovell MR, Kegel NE, et al:: The relationship of symptoms and neurocognitive performance to perceived recovery from sports-related concussion among adolescent athletes. Appl Neuropsychol Child. 2013; 2(1): 64-9.

PubMed Abstract | Publisher Full Text

4. Goodbody SJ, Wolpert DM: Temporal and amplitude generalization in motor learning. J Neurophysiol. 1998; 79(4): 1825-38. PubMed Abstract

5. Wang J, Sainburg RL: Adaptation to visuomotor rotations remaps movement vectors, not final positions. J Neurosci. 2005; 25(16): 4024-30. PubMed Abstract | Publisher Full Text

6. Kagerer FA, Bo J, Contreras-Vidal JL, et al:: Visuomotor adaptation in children with developmental coordination disorder. Motor Control. 2004; 8(4): 450-60. PubMed Abstract
7. Contreras-Vidal JL, Bo J, Boudreau JP, et al:: Development of visuomotor representations for hand movement in young children. Exp Brain Res. 2005; 162(2): 155-64.

PubMed Abstract | Publisher Full Text

8. Tombaugh TN: Trail making test A and B: normative data stratified by age and education. Arch Clin Neuropsychol. 2004; 19(2): 203-14.

PubMed Abstract | Publisher Full Text

9. Bowie CR, Harvey PD: Administration and interpretation of the Trail making Test. Nat Protoc. 2006; 1(5): 2277-81. PubMed Abstract | Publisher Full Text

10. Gaudino EA, Geisler MW, Squires NK: Construct validity in the Trail Making Test: what makes Part B harder? J Clin Exp Neuropsychol. 1995; 17(4): 529-535.

PubMed Abstract | Publisher Full Text

11. Lezak MD, Howieson DB, Loring DW: Neuropsychological Assessment. 4th ed. New York: Oxford University Press. 2004. Reference Source

12. Wang J, Thomas D, Cho YI: Dataset 1 in: Sensorimotor learning deficits observed in children with sports-related concussion. F1000Research. 2015. Data Source 


\section{Open Peer Review}

\section{Current Peer Review Status: $\mathrm{X}$}

\section{Version 1}

Reviewer Report 24 June 2015

https://doi.org/10.5256/f1000research.7060.r8919

(C) 2015 Sergio L. This is an open access peer review report distributed under the terms of the Creative Commons Attribution License, which permits unrestricted use, distribution, and reproduction in any medium, provided the original work is properly cited.

\section{Lauren Sergio}

Center for Vision Research, York University, Toronto, ON, USA

This study describes a psychophysical experiment in which three children who had recently experienced a concussion were asked to perform a visuomotor adaptation. Their performance was compared to three children with no concussion history. While the motivation for the study was sound, I have a number of concerns with this manuscript:

1. Introduction: "However, the association between pediatric concussion and sensorimotor function has not been investigated." An inaccurate statement, de Beaumont has done some work in this area, as has Danckert (review references listed at end).

2. Methods: Won't Measure 2 always co-vary with Measure 3? Perhaps I am misunderstanding something. The rate of adaptation should always be related to the point at which they reach baseline performance, no?

3. Major concern: Given the variety in symptoms and behavioural response to concussion, a study using only 3 children should be considered along the lines of a case study, or extremely preliminary data at least, more suitable for an abstract. Minimally, it should be made clear in the intro that this is a case study (only mentioned at end of methods). In general, this type of study is not appropriate as a case study since adolescent concussion is not, unfortunately, a rare or unusual situation. It would not be overly cumbersome to have an appropriate sample size (10-12 each group), fully analyse the data, and only then report the findings.

4. It should be noted if this was the participants' first concussion or not, since this is often an important distinguishing variable in post-concussion recovery rate. It should also be noted if the experimental group members were still symptomatic (via something like a SCAT3).

5. Major concern: Given the significant neurocognitive and neurological development between 12 and 15, three age-matched controls would have been better. Also, because of sex-related differences in visuomotor control (see Gorbet et al. 2007, 2011) and sex-related developmental differences in this age range, the sex of the children should be stated and 
matched with controls.

6. Typically this paradigm includes a 'washout' phase whereby participants do a number of trials in a null field. The level of aftereffect serves as an indication of the extent to which the adaptation was incorporated. This might be useful for the present design.

7. TMT result: Again, there is the issue of comparing a 12 year old to 15 year olds to a 17 year old, so it is difficult to judge whether this is an age effect or a concussion effect. Age is typically a strong covariate for coordination measures taken pre/post-concussion (see Dalecki et al. 2015).

8. It appears that the worse the concussed participant did on the TMT, the later the point of adaption-baseline match (cc1 and cc3 vs cc2). This is something which cannot be explored

\section{Conclusion:} with $n=3$, but should be noted and examined with the larger data set.

The concept and approach are interesting and potentially useful, and indeed the use of sensorimotor impairment in the absence of current-standard symptoms post-concussion is an important area of study. However, the inappropriate presentation as a case study and the lack of age (and sex) controls make it difficult to consider this a full and rigorous study. In its present form it is suitable as a presentation of preliminary data at a conference rather than a cite-able publication. I look forward to seeing the complete study.

\section{Minor comments:}

1. Methods, paragraph 1: grammar issue (tense change) - "Selection criteria for subjects were the same between patients and controls (except their concussion status), which were: subject is 10-17 years of age,regularly participates in an athletic activity, is English-speaking, is right handed, and has no neurological disease or peripheral disorder affecting movement of the right arm."

2. Figure 1a: Is the axis mislabeled for figure $1 \mathrm{a}$ (left)? The methods state that there are 40 baseline trials.

3. Figure $1 \mathrm{~b}$ : consider using a different symbol shape for controls, difficult to distinguish open versus filled circles.

4. Figure caption error (two 'd' references, no ' $f$ ' reference)

\section{References:}

De Beaumont L, Tremblay S, Henry LC, et al. Motor system alterations in retired former athletes: the role of aging and concussion history. BMC Neurol. 2013;13:109.

Locklin J1, Bunn L, Roy E, Danckert J. Measuring deficits in visually guided action post-concussion. Sports Med. 2010 Mar 1;40(3):183-7.

Gorbet DJ, Sergio LE. Preliminary sex differences in human cortical BOLD fMRI activity during the preparation of increasingly complex visually guided movements. Eur J Neurosci. 2007;25:1228- 
1239.

Gorbet DJ, Staines WR. Inhibition of contralateral premotor cortex delays visually guided reaching movements in men but not in women. Exp Brain Res. 2011 Jul;212(2):315-25.

Dalecki MS, Sergio LE. Prolonged cognitive-motor impairments in children with a history of concussion. \#2-D-107, Canadian Association for Neuroscience, 2015, Vancouver, BC. http://canacn.org/documents/2015/CAN2015_Abstract_Booklet.pdf

Competing Interests: No competing interests were disclosed.

\section{I confirm that I have read this submission and believe that I have an appropriate level of expertise to state that I do not consider it to be of an acceptable scientific standard, for reasons outlined above.}

Author Response 17 Jul 2015

Jinsung Wang, University of Wisconsin- Milwaukee, Milwaukee, USA

Thank you for your review. In our responses below, we have attempted to address each concern raised by you.

Introduction: "However, the association between pediatric concussion and sensorimotor function has not been investigated." An inaccurate statement, de Beaumont has done some work in this area, as has Danckert (review references listed at end):

We believe this statement by the reviewer is incorrect, in that neither study investigated association between concussion and sensorimotor function in children. de Beaumont et al. investigated a motor learning issue in retired athletes aged between 51 and 75 years; and Danckert and colleagues investigated a motor control issue in young adults aged between 17 and 27 years.

Methods: Won't Measure 2 always co-vary with Measure 3? Perhaps I am misunderstanding something. The rate of adaptation should always be related to the point at which they reach baseline performance, no?:

Our measures 2 and 3 do not necessarily co-vary with each other, especially given that measure 3 is influenced by the size of direction error at the beginning of adaptation whereas measure 2 is not.

Major concern: Given the variety in symptoms and behavioural response to concussion, a study using only 3 children should be considered along the lines of a case study, or extremely preliminary data at least, more suitable for an abstract. Minimally, it should be made clear in the intro that this is a case study (only mentioned at end of methods):

As the reviewer noted, this research note reports movement data obtained from a very small number of children. In fact, we originally intended to publish this short manuscript as a clinical practice article. According to the journal's guidelines, clinical practice articles include series of case reports, which do not need to describe especially novel or unusual cases. During the in-house check, however, the editor decided that our manuscript would be more suitable as a research note. We have now modified the introduction section to emphasize the nature of this study (i.e., observational/case study). 
It should be noted if this was the participants' first concussion or not, since this is often an important distinguishing variable in post-concussion recovery rate. It should also be noted if the experimental group members were still symptomatic (via something like a SCAT3):

Our patients never had a concussion prior to this one. We did not assess their symptoms on the day they participated in the experiment, although one of the patients (cc1) was not able to speak or walk normally at that time. We have included this information in the methods section now.

Major concern: Given the significant neurocognitive and neurological development between 12 and 15, three age-matched controls would have been better. Also, because of sex-related differences in visuomotor control (see Gorbet et al. 2007, 2011) and sex-related developmental differences in this age range, the sex of the children should be stated and matched with controls:

We agree that it would have been better if both age and sex were matched perfectly between the patients and the controls. However, we are not aware of any findings that indicate significant differences between boys and girls within the age range of 12-17 years with regard to their visuomotor adaptation capabilities. There is at least some evidence that visuomotor representations used by children older than 11 years are similar to those used by adults. For example, Ferrel et al. (2001, Exp Brain Res) suggested that during targeted reaching movements under a visuomotor rotation condition, visuomotor representations of children who were 6 or 8 years old differed from those of adults, although those of 11 yearold children did not. In addition, our data do not seem to indicate sex-related differences clearly. For example, the adaptation pattern of cc2 (15 year-old female) appears to be very similar to that of ctls 1 and 3 ( 12 and 14 year-old males, respectively) in terms of our measures 2 and 3 . Thus, it seems unlikely that our results were substantially influenced by age- or sex-related differences among the participants. Nonetheless, we agree that we cannot completely exclude the possibility that age and sex of the participants played some role. Therefore, we have now included additional statements in the discussion section to address this point. We have also provided sex information of our participants in the methods section.

Typically this paradigm includes a 'washout' phase whereby participants do a number of trials in a null field. The level of aftereffect serves as an indication of the extent to which the adaptation was incorporated. This might be useful for the present design: We did not include a washout phase in our present design, but we plan to do so in the future when we can conduct a more rigorous study with a larger sample size.

TMT result: Again, there is the issue of comparing a 12 year old to 15 year olds to a 17 year old, so it is difficult to judge whether this is an age effect or a concussion effect. Age is typically a strong covariate for coordination measures taken pre/postconcussion (see Dalecki et al. 2015):

The main result of the present study in terms of TMT is that the TMT scores of all pediatric patients tested in our study (including cc1 whose score was the largest) were below 100 seconds, which is substantially better than the score that is considered deficient (i.e., 273 seconds). Also, our data indicate that the TMT scores of all our subjects except cc1 (15 yearold female) range only from 27 to 53 seconds, which do not appear to vary depending on the subject's age or sex. Thus, it seems unlikely that our TMT result was influenced substantially by the subject's age.

It appears that the worse the concussed participant did on the TMT, the later the 
point of adaption-baseline match (cc1 and cc3 vs cc2). This is something which cannot

be explored with $n=3$, but should be noted and examined with the larger data set:

We agree that this is something that should be examined (when one has a larger data set). However, we would like to point out that the TMT score of cc3 fell within the range of the TMT scores obtained from the controls and that his score was substantially better than the score considered deficient, which indicates that this patient did not suffer from any cognitive slowing. Given that, our data do not suggest a strong association between TMT performance and visuomotor adaptation at least in the concussed children who were tested in our study.

\section{Minor comments:}

Methods, paragraph 1: grammar issue (tense change) - "Selection criteria for subjects were the same between patients and controls (except their concussion status), which were: subject is 10-17 years of age, regularly participates in an athletic activity, is English-speaking, is right handed, and has no neurological disease or peripheral disorder affecting movement of the right arm.":

We have changed the tense now.

Figure 1a: Is the axis mislabeled for figure $1 \mathrm{a}$ (left)? The methods state that there are 40 baseline trials:

The reviewer is correct that there were 40 baseline trials. We have corrected this error now. Figure $1 \mathrm{~b}$ : consider using a different symbol shape for controls, difficult to distinguish open versus filled circles:

We have now included the lines that are either thick or thin to help distinguish between the patients and controls.

Figure caption error (two ' $d$ ' references, no ' $f$ ' reference):

We have corrected this error now.

Competing Interests: None 
The benefits of publishing with F1000Research:

- Your article is published within days, with no editorial bias

- You can publish traditional articles, null/negative results, case reports, data notes and more

- The peer review process is transparent and collaborative

- Your article is indexed in PubMed after passing peer review

- Dedicated customer support at every stage

For pre-submission enquiries, contact research@f1000.com 